sures would remain to be elucidated.

Such an experiment may confirm Ryan et al.'s hypothesis that female preference has not evolved at all, but sensory exploitation alone cannot explain the evolution of either the male call or the maintenance of female preference. The male chuck is notable because its mean frequency is higher than the peak female receptivity; a signal evolved solely through sensory exploitation should coincide with peak receptivity, not fall short of it. It seems likely to us that the shortfall in the male chuck is the result of counter-selection against lower frequency calling (perhaps deep calls are costly), implying that processes other than sensory exploitation are important. Alternatively, but less plausibly, there may be some genetic constraint on the evolution of deeper chucks.

For these reasons, we think it unlikely that sensory exploitation can offer a genuinely alternative explanation to that of the evolution of female preference through sexual selection. In a sense, all signals must evolve along routes that exploit sensory biases in the signal receiver. The crucial question is not whether sensory exploitation has been involved, but why females have allowed themselves to be exploited. Ryan et al. suggest this is because mate preference is fixed, presumably by genetic constraints, yet there is plenty of evidence that preferences can evolve in anurans $s^{2,3}$. We believe it is far more likely that females benefit through their mate preference because properties of the male chuck (such as frequency) contain useful information about male quality. If this were not the case - if, for example, sensory bias led females to prefer infertile males - then selection would act to extinguish such a preference.

Indeed, Ryan et al. themselves report one direct advantage of preference: females have more fertilized eggs when they mate with larger males and larger males have lower frequency chucks. There may also be other heritable benefits, such as those proposed in the DarwinFisher (attractive sons) or the 'good genes' (high offspring survival) views of sexual selection. Neither of these hypotheses can be ruled out by the reported data. Sensory exploitation is not a rival of these hypotheses. The evolution of signals and their reception will occur not ony through sensory exploitation, but also through selection on the signal receiver and signal producer.

ANDREW POMIANKOWSKI TIM GUILFORD

\section{Department of Zoology,}

University of Oxford,

Oxford OX1 3PS, UK

1. Ryan, M.J. et al. Nature 343, 66-67 (1990).

2. Hoy, R.H., Hahn, J. \& Paul, R.C. Science 195, 82-84 (1977).

3. Ryan, M.J. and Wilczynski, W. Science 240, 1786-1788 (1989)

\title{
Superficial microwave heating
}

SIR-The domestic market for microwave $\mid$ meals investigated were 0.024-0.15 M ovens has grown rapidly in Britain since

sodium (mean $0.102 \mathrm{M}$ ); $0.012-0.12 \mathrm{M}$

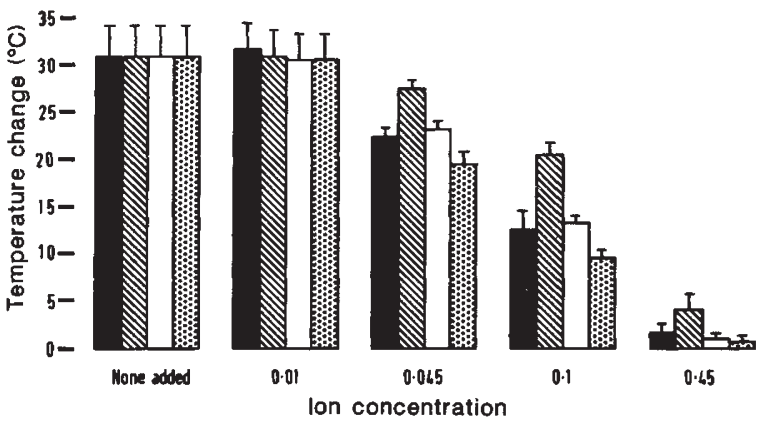

The temperature change induced at the core of $200 \mathrm{~g}$ mashed potato by a $650-W$ microwave oven in 1 minute versus the concentration of ionic compounds present in the food. The ranges indicate one standard deviation. Filled columns, $\mathrm{NaCl}$; striped, monosodium glutamate; open, $\mathrm{NH}_{4} \mathrm{Cl}$; stippled, $\mathrm{KCl}$.

the early 1980 s, helped by advertising slogans such as "heats from the inside out". But there were no scientific data to support these claims. The recent increase in salmonellosis and listeriosis has suggested that the bacteria causing these diseases, if present in food ${ }^{1-3}$, are not being killed by reheating or cooking. We have measured the change in temperature of food as a measure of energy transfer to it from microwaves and have found that such temperatures are not adequate reliably to kill bacteria present ${ }^{4}$. We wondered whether the apparent contradiction could be caused by high ionic concentrations in the food, allowing the induction of ionic currents by the microwaves and causing energy to be released, preventing the microwaves from penetrating the food to heat the core.

We made mashed potato with no added salts, measured its ionic content and cooled it to $8^{\circ} \mathrm{C}$. We added dissolved chemical salts $\left(\mathrm{NaCl}, \mathrm{KCl}, \mathrm{NH}_{4} \mathrm{Cl}\right.$ and monosodium glutamate) to some samples. Each sample was put onto the centre of the rotating turntable of a Matsui $170 \mathrm{TC}$ $650-\mathrm{W}, 1250-\mathrm{MHz}$ domestic microwave oven and heated for $1 \mathrm{~min}$. The core temperature was measured directly after heating. We repeated the experiments using $300 \mathrm{~g}$ mashed potato in a cook-chill microwave oven, and we also investigated some ready-prepared meals.

The core temperature change of the mashed potato in the beaker decreases with increasing concentration of the added salts (see figure). In the microwave oven dish, the temperature change of the food with added salts was about 62 per cent of that when no salts were added. The concentrations of sodium and potassium in the 10 commercial ready-processed

1. Lancet 231, 720-722 (1988).

2. Gellin, B.G. \& Broom, C.V. J. med. Ass. Am. 261, 13131320 (1989).

3. Rampling, A. et al. Lancet 334, 436-438 (1989)

4. Sheeran, M.R.M. et al. J. hosp. Infec. 14, 84-86 (1989) potassium (mean 0.036 M). These concentrations are clearly in the range that would be affected.

The poor penetration of microwaves into the test food with high ionic concentrations may result from the induction of electrical/ionic flow in the surface of the food. This would also explain why commercial food heated in microwaves commonly boils on the surface but is cool on the inside.

StePHen F. DEALleR RICHARD W. LACEY Department of Medical Microbiology, Leeds University, Leeds LS2 9JT, UK

\section{Irradiated cells}

SIR-The association of childhood leukaemias and fathers' occupational exposure to radiation reported by Gardner $e t a l .{ }^{1}$ raises the possibility that if workers were contaminated with unidentified radionuclides, which may be enriched in the urogenital organs or the semen, these would not be detected by external dosimeters.

An objective of the late Radiological Protection Service MRC Stable Element Unit was to determine the abundances of elements in human tissues, organs and their routes of entry in relation to those for radioactive analogues ${ }^{2}$. For the radionuclides of the heavy alpha-emitting radionuclides, which are often associated with small volumes or surfaces, suitable stable-element analogues are the rareearth elements and possibly bariumsulphur pathways. Rare-earth elements have been detected in bone, liver, kidney, lymph nodes and testis, but not in ovaries, using autopsy samples following accidental deaths. Wester ${ }^{3}$, using neutronactivation analysis, also found cerium and lanthanum in RNA and the sacrotubular fraction of beef heart tissue.

If a single-hit hypothesis for the induction of a cancer by alpha-emitters is accepted, then it seems likely that damage to spermatogonia or their stem cells is possible. Today the concept of multiple hits is not considered likely, even in the instance of irradiation of cells by 'hot' particles for which high concentrations of radionuclides are associated with small volumes. Nevertheless, the subcellular distribution, or surface enrichment, of 